\title{
Dystocia in a Rothschild Giraffe at the African Fund for Endangered Wildlife, Nairobi, Kenya
}

\author{
Kaitho T D*, Limo C K, Rono B, Ndambiri E M \\ Veterinary and Capture Services Department, \\ Biodiversity Research and Monitoring Division, Kenya Wildlife Service, \\ Langata Road, P. O. Box 40241-00100, Nairobi, Kenya \\ *Corresponding author email: titus@kws.go.ke, Tel. +254728932369 \\ Received: 5-7-2011, Accepted: 13-07-2011, Published Online: 23-10-2011 \\ doi: $10.5455 /$ vetworld.2011.565-568
}

\begin{abstract}
A 15-year old female Rothschild Giraffe (Giraffa camelopardalis rothschildi) weighing approximately $800 \mathrm{~kg}$, at the African Fund for Endangered Wildlife (AFEW), Giraffe Center, Langata, Nairobi, Kenya was presented with dystocia in June 2010. This giraffe named Laura, had a protracted labor and was regularly monitored by sanctuary education staff. Dystocia was relieved on the $3^{\text {rd }}$ day at this wildlife sanctuary. The giraffe was chemically immobilized by using $7 \mathrm{mg}$ of Etorphine $\mathrm{Hcl}(0.98 \%)\left(\mathrm{M}^{\circledR} 9^{\circledR}\right.$ ) (Norvatis South Africa (Pty) Limited) and 50mg of Azaperone(10\%) (Kyron Laboratories (Pty) Limited, South Africa) in a Dan-Inject dart (Dan-inject APS, Sellerup Skowej, Denmark). On obstetrical examination of the giraffe, a fetal malposition type of dystocia had occurred. The fetus was positioned at posterior presentation extended posture with tail butting on the maternal pelvis, which is abnormal in giraffes. The fetus was manually extracted by using both alternate and simultaneous limb traction. The dam survived the procedure and later was reported to be in a good reproductive condition but the male fetus was a stillbirth. The fetus had died due to stress of prolonged labour. Relief of dystocia in giraffes is a difficult obstetrical procedure because obstetrical examination and relief requires chemical immobilization plus physical restrain with ropes by trained staff. Anesthesia or immobilization of giraffes remains a challenge because of the giraffe's unique anatomy and physiology. Giraffes are large animals which limits physical control and manipulation at critical times during induction and recovery of anesthesia. Giraffe's long neck if not pinned to the ground will act as a lever causing fatal injuries to self and support staff. Giraffes develop elevated systolic blood pressure; have a small respiratory tidal volume with a large dead space and relatively small cardiac output during anesthesia, which compromises safe levels of anesthesia.
\end{abstract}

Key words: Giraffe, dystocia, manual extraction

\section{I ntroduction}

The African Fund for Endangered Wildlife (AFEW), Giraffe Center, Nairobi manages a herd of Rothschild giraffes which are categorized as endangered in the International Union for Conservation of Nature (IUCN) red list of threatened species (Fennessy and Brennema 2010). The giraffes roam freely in a 100 acre fenced wildlife sanctuary in the suburban area of Nairobi. This herd of giraffe is sometimes fed with supplements and is habituated to visitors who occasionally try to feed them by hand. On the contrary these giraffes remain wild and aggressive and can kick if sloppily approached.

Successful management of dystocia in giraffes with survival of the mother and the baby giraffe is a rare occurrence. Lack of a standard facility and equipment for a cesarean section in the field conditions and inherent anesthetic risk due to giraffe's anatomy and physiology further complicates management of dystocia in the species. Dystocia in giraffes has been documented previously by some authors (Ebedes, 1975, Citino et al., 1984, Goslawki and Kolodziejska 1984). Survival of the mother giraffe after caesarian operation and a dead fetus has also been reported by Williams et al. (2007). Relief of dystocia in a zoo facility by zoo staff using rope snares when a dam was under no anesthesia has been reported by Hwan-Yul Yong et al., (2009).

Relief of dystocia in a giraffe kept in a wildlife sanctuary by manipulation and traction under anesthesia with favorable outcome has not been reported. A single case of the same has been successfully handled by Kenya Wildlife Service staff at Soysambu ranch, Nakuru (Gakuya 2003). 


\section{Materials and methods}

Case clinical progression: On $25^{\text {th }}$ June 2010 a 15 year old giraffe named Laura, from this sanctuary was reported to have shown signs of labour with no fetal membranes or parts seen. Blood tinged exudates were sighted on the ventral commisure of the vulva. She was straining, had decreased appetite and looked restless, anxious and isolated herself from the herd. On the $2^{\text {nd }}$ day fetal membranes about $30 \mathrm{~cm}$ long and fetal legs were seen hanging from the vulva. On $28^{\text {th }}$ June 2010 there was no further progress and a decision was made to restrain the animal for obstetric examination and management.

Restraint: The giraffe was darted using $7 \mathrm{mg}$ of $0.98 \%$ Etorphine $\mathrm{Hcl}\left(\mathrm{M} 9^{\circledR}\right.$ ) (Norvatis South Africa (Pty) Limited) and 50mg of $10 \%$ Azaperone $\mathrm{Hcl}$ (Kyron Laboratories (Pty) Limited, South Africa) in a Dan-Inject dart (Dan-inject APS, Sellerup Skowej, Denmark) on the shoulder muscles. The animal was darted on foot. Induction time was 10 minutes. During induction to down time, the animal ran into the bushes which posed a great challenge. Anesthesia was partially reversed immediately with $21 \mathrm{mg}$ of $1.2 \%$ Diprenorphine $\mathrm{Hcl}$ (Norvatis South Africa (Pty) Limited) given $1 / 4$ of the dose intravenously in the jugular vein and restrained physically using ropes. A blindfold was used to minimize stress, Opticlox® eye ointment (Norbrook laboratories Ltd) applied on the both eyes to prevent corneal drying and laceration. Clearing of the bushes was quickly carried out to facilitate access and handling of the giraffe. Complete reversal of anesthesia was achieved by using the balance of $15 \mathrm{mg}$ Diprenorphine administered intravenously and 50mg of 5\% Naltrexone (Kyron Laboratories (Pty) Limited) injected intramuscularly to prevent re-narcotization.

Obstetrical examination and case management: Obstetrical examination revealed dead fetus on

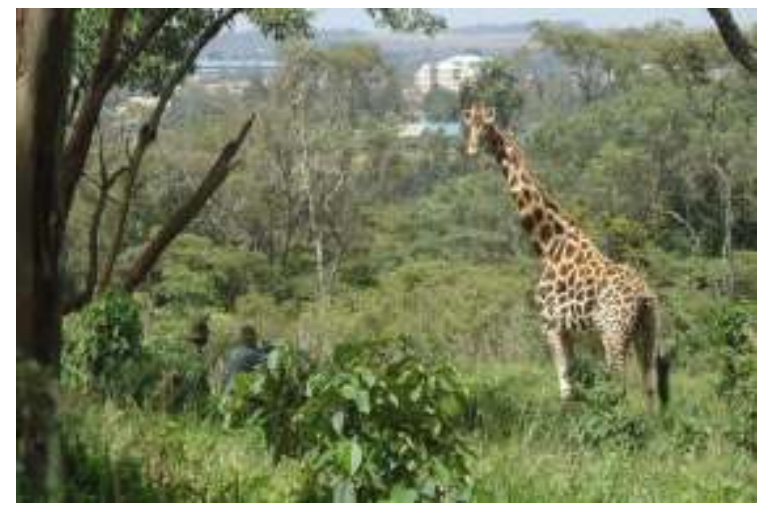

Figure-1: Fetal legs sighted posterior presentation with an extended posture. The tail was also butting on the pelvic brim (figure 1 and 2).Type of dystocia was a fetal malposition(fetal malpresentation) - a posterior presentation with an extended posture.

The fetus was retro-pulsed and realigned. Fetal skin was sloughing off. Manual extraction and manipulation of the fetus with the aid of ropes and liberal lubrication using liquid paraffin was carried out (figure 3 and 4). Fetal membranes were removed manually and Betamox ® LA (Amoxicillin 15000mg) (Norbrook laboratories Ltd) administered intramuscularly. Samples were taken including placental caruncles for Brucella culture.

\section{Discussion}

Wildlife clinical interventions are guided by sound principles governed by animal welfare and safety to staff. The right time to intervene in a dystocia case in relation to safety of the dam, baby giraffe and not the least staff, is critical in achieving a favorable outcome. Citino et al. (1984) has recommended that obstetrical examination be carried out after two hours of active parturition without noticeable signs of progress. Dagg and Foster (1982) supported this and stated that a giraffe calving should last between one and two hours. On the contrary Kristall and Noonan (1979) reported a parturition lasting approximately 15 hours, with no fetal protrusion shown during the first 14 hours but a healthy calf being born uneventfully. The practicability of intervention is dependent on the facilities available and other factors need to be considered (Williams et al., 2007). Williams et al.(2007) has observed that if physical restraint of a giraffe is possible thus allowing for obstetrical examination to be carried out safely, then it is preferable to intervene sooner than later. Nevertheless if an examination is only possible through the use of

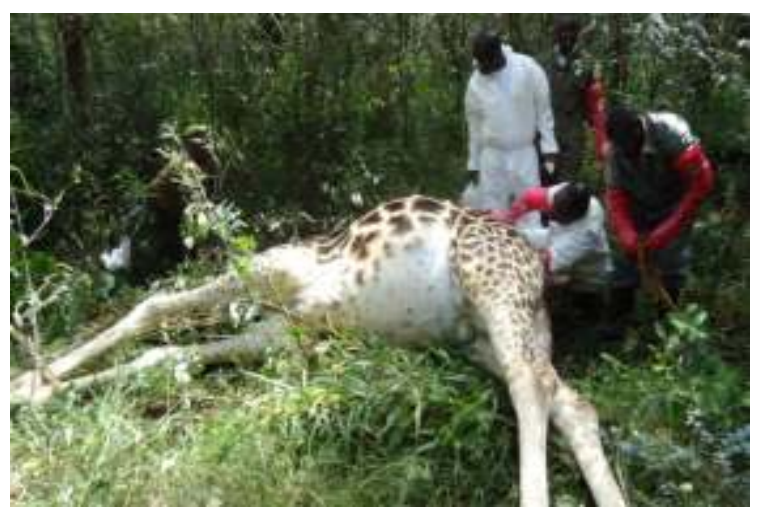

Figure-2: Obstetrical examination 


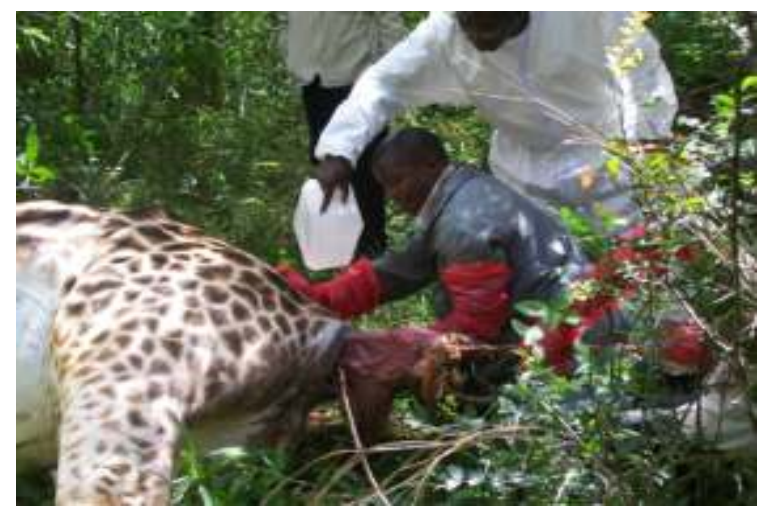

Figure-3: Manipulation of fetus with liberal lubrication

chemical immobilization, then it may seem not preferable to rush to intervene.

According to Bush et al. (2002) giraffe's distinctive anatomy and physiology as described by Dagg and Foster (1976), presents inherent problems during anesthesia or chemical restraint include the following:

1. Their large size limits physical control during critical times of induction and recovery, and limits manipulation once the animal is down.

2. Their characteristic long neck, which if not controlled, acts as a lever arm creating danger to itself or the support staff. Solounias (1999) has proposed that the giraffe has actually eight cervical vertebrae contrary to the popular observation that all mammals have 7 cervical vertebrae. Mal-positioned neck leads to air way obstruction which is fatal. Burroughs et al., (2006) have reported whip lash effect of the long neck resulting in head trauma. In addition to this the long hind and front legs can pose a danger to staff.

3. Improper choice of anesthetic drugs potentiates self induced injury due to slipping during induction and recovery.

4. Their tendency to vomit or regurgitate can lead to fatal aspiration pneumonia and the posterior position of the larynx in the pharynx hampers draining of any fluid (rumen and/or saliva).

5. Prolonged induction and/ or recovery leads to hyperthermia, myopathy, and secondary trauma. Giraffes usually develop elevated systolic blood pressure, has a small respiratory tidal volume with a large dead space (Hugh-Jones et al., (1979) and relatively small cardiac output during anesthesia.

Posterior presentation (fetal hind extremity directed towards the maternal pelvis) is considered

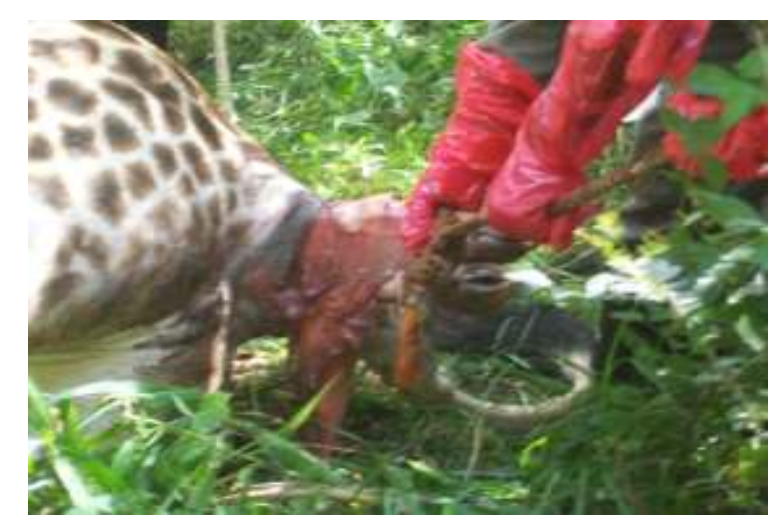

Figure-4: Traction using ropes

(Benesch and Wright 1957) as abnormal in uniparous domestic species. More space is required for the hind limbs to undergo full extension than is the case with the fore. Therefore the longer the limbs the more complications can be expected (Devos, and Bourquin 1974). No other land mammal equals the size of the giraffe at birth (Hediger and Klages 1968). This is mainly due to the typically long legged build and elongated neck of the species. Posterior presentation must therefore be considered as abnormal in a giraffe. Prolonged labour may have resulted in fetal death and exhaustion of the dam. To rule out any infection a sample of fetal membranes containing caruncles was collected for culture.

The procedure took 20 minutes and the animal was successfully revived with good prognosis for survival and future breeding. This in our opinion contributed much to the favorable outcome. Our anesthesia protocol and duration was sound and practical. Vogelnest and Ralph (1997) reported similar protocols for giraffes to facilitate short procedures. The manual extraction of calves in cases of dystocia is a technique performed in daily practice, but there are divergent recommendations concerning the procedure (Mee 2004, Noakes 2001, Norman and Youngquist 2007).

Berker et al. (2010) have objectively supported the pulling of the elbows one at a time into the pelvic cavity and then extracting the fetus by applying traction to both limbs simultaneously in anterior presented bovine calves. This method of extraction keeps the traction forces to a minimum. We used alternate limb traction and later simultaneous limb traction with lots of lubrication which in our considered opinion works well. Tsousis et al. (2011) have carried an intensive study in extraction methods in bovine obstetrics. Giraffes remain delicate animals as they can easily succumb to self inflicted injuries, 
and complications during and after anesthesia.

\section{Acknowledgements}

The authors would like to thank Kenya Wildlife Service capture rangers, The African Fund for Endangered Wildlife (AFEW) - Giraffe Center team and Dr Derrick Chibeu of Sercombes Veterinary clinic for a job well done.

\section{References}

1. Becker, M, Tsousis G, Lüpke, M, Goblet, F, Heun, C, Seifert, H and Bollwein, H (2010). Extraction forces in bovine obstetrics: An in vitro study investigating alternate and simultaneous traction modes. Theriogenology.73:10441050.

2. Benesch, F and Wright, JG (1957). Veterinary Obstetrics. London: Bailliere, Tindall and Cox.

3. Burroughs, R, Morkel, P, Kock, MD, Meltzer, D and Hofmeyr, M (2006). Chemical immobilization. In Chemical and physical restraint of wild animals - a training and field manual for African species. Eds Kock MD, Meltzer D and Burroughs R. IWVS, Greyton, South Africa.

4. Bush, M, Grobler, DG and Raath, JP (2002). The art and science of giraffe (giraffe camelopardalis) immobilization / anesthesia. In: Zoological Restraint and Anesthesia, D. Heard(Ed), International Veterinary Information service (IVIS) (www.ivis.org) Ithaca, New York USA.

5. Citino, SB, Bush, M and Phillips, LG (1984). Dystocia and fatal hyperthermia episode in a giraffe. Journal of American Veterinary Medicine Association, 1440-1442.

6. Dagg, AI and Foster, JB (1976). Anatomy and physiology of the viscera (Appendix B). In: The giraffe: its biology, behavior and ecology. New York: Van Nostrand Company, Pp160-170.

7. Dagg, AI and Foster, JB (1982). The giraffe: Its Biology Behavior and ecology. Krieger Publishing Company, Melbourne, Fla, USA.

8. Devos, V and Bourquin, O (1974). Dystocia in a giraffe (giraffe camelopardalis). Koedoe 17:199-203.

9. Ebedes, H (1975). Dystocia in free roaming giraffe (Giraffa camelopardalis). Journal of south African Veterinary Association. 46, 287.

10. Fennessy, J and Brenneman, R (2010). Giraffa camelopardalis ssp. rothschildi. In: IUCN 2010. IUCN Red List of Threatened Species. Version 2010.4. <www. iucnredlist.org> Downloaded on 21 February 2011.

11. Gakuya, F (2003). Relief of dystocia in Rothschild giraffe in Soysambu Ranch, Nakuru. Unpublished field case report of $10^{\text {th }}$ May 2003.

12. Goslawki, J and Kolodziejska, Z (1984). Dystocia of a giraffe and a attempt to raise newborn on cow colostrum. Int. symp. Über die Erkrankungen Zootiere 26, 125-129.

13. Hidiger, H and Klages, J (1968). Born in the zoo. London: Collin. St. James's palace.

14. Hugh-Jones, P, Barter, CE, Hime, JM, et al.(1978). Dead space and tidal volume of the giraffe compared with other mammals. Respiratory Physiology, 35:53-58.

15. Hwan-Yul Yong, Suk Hyun Park, Myoung-Keun Choi, SoYoung Jung, Dae-Chang Ku, Jong-Tae Yoo, Mi-Jin Yoo,MiHyun Yoo, Kyung-Yeon Eo, Yong-Gu Yeo, Shin-Keun Kang and Heon-Youl Kim (2009). Baby giraffe pulled out of mother suffering from dystocia without proper restraint device. Journal of Veterinary Clinics 26(1):113-116.

16. Kristal, MB and Noonan, M (1979). Prenatal maternal and neonatal behavior in the captive reticulated giraffe. South African Journal of zoology 14, 103-107.

17. Mee, JF (2004). Managing the dairy cow at calving time. Veterinary clinics of North America Food Animal Practice; 20:521-46.

18. Noakes, DE (2001). Dystocia due to fetomartenal disproportion; Treatment. In Authur's Veterinary Reproduction and Obstetrics $8^{\text {th }}$ edition, Noakes, DE, Parkison, TJ and England, GCW (Eds)W.B.Saunders, Pp279-89.

19. Norman, S and Youngquist, RS (2007). Parturition and dystocia. in: Current therapy in large animal theriogenology $2^{\text {nd }}$ edition Youngquist, RS, Threlfall, WR (Eds) Saunders, Pp310-35

20. Solounias, N (1999). The remarkable anatomy of the giraffe's neck. Journal of Zoological Society of London 247, 257268.

21. Tsousis, G, Becker, M, Lüpke, M, Goblet, C, Heun, H, Seifert, $\mathrm{H}$ and Bollwein, $\mathrm{H}$ (2011). Extraction methods in bovine obstetrics: A comparison of the demand energy and importance of calf and traction method in the variance of force and energy. Theriogenology 75: 495-499.

22. Vogelnest, L and Ralph, HK 1997. Chemical immobilisation of giraffe to facilitate short procedures. Australian Veterinary Journal 75(3): 180-182.

23. Williams, DC, Murison, PJ and Hill, CL (2007). Dystocia in a Rothschild giraffe leading to a caesarian section. Journal of Veterinary Medicine A. 55:199-202. 